\title{
THE ORDER BIDUAL OF ALMOST $F$-ALGEBRAS AND $D$-ALGEBRAS
}

\author{
S. J. BERNAU AND C. B. HUIJSMANS
}

\begin{abstract}
It is shown in this paper that the second order dual $A^{\prime \prime}$ of an Archimedean (almost) $f$-algebra $A$, equipped with the Arens multiplication, is again an (almost) $f$-algebra. Also, the order continuous bidual $\left(A^{\prime}\right)_{n}^{\prime}$ of an Archimedean $d$-algebra $A$ is a $d$-algebra. Moreover, if the $d$-algebra $A$ is commutative or has positive squares, then $A^{\prime \prime}$ is again a $d$-algebra.
\end{abstract}

\section{INTRODUCTION, PRELIMINARIES}

In this paper we will consider the second order dual $A^{\prime \prime}$ of an Archimedean lattice ordered algebra $A$, equipped with the so-called Arens multiplication, in great detail. Our main results will be that the order bidual $A^{\prime \prime}$ of an Archimedean (almost) $f$-algebra is again an (almost) $f$-algebra and that the order continuous order bidual $\left(A^{\prime}\right)_{n}^{\prime}$ of an Archimedean $d$-algebra is equally an Archimedean $d$-algebra. The question of whether in the latter case the whole second order dual $A^{\prime \prime}$ is a $d$-algebra remains open.

In order to avoid unnecessary repetition we will assume throughout that all vector lattices and lattice ordered algebras under consideration are Archimedean.

Let us recall some of the relevant notions. For terminology and results on vector lattices and $l$-algebras not explained in this paper we refer to [1], [9], [10], and [14]. Let $A$ be a (real) vector lattice (or Riesz space). The first order dual of $A$ is denoted by $A^{\prime}$ and the second order dual by $A^{\prime \prime}$. It is well known that $A^{\prime}$ is a Dedekind complete (and hence Archimedean) vector lattice. Define for each $x \in A$ the element $x^{\prime \prime} \in A^{\prime \prime}$ by $x^{\prime \prime}(f)=f(x)$ for all $f \in A^{\prime}$, and then define the mapping $\sigma: A \rightarrow A^{\prime \prime}$ by $\sigma(x)=x^{\prime \prime}$ for all $x \in A$. We can easily check that $\sigma$ is a vector lattice homomorphism of $A$ into $A^{\prime \prime}$ ([14, $\S 109]$ ). It is possible that $A^{\prime}$ is trivial (see for example [14, Examples 85.1 and 85.2]). However, if $A^{\prime}$ separates the points of $A$, then $\sigma$ is injective and we can identify $A$ with the vector sublattice $\sigma(A)$ of $A^{\prime \prime}$. Clearly $A^{\prime \prime}$ is Dedekind complete (and, of course, trivial if $A^{\prime}$ is trivial). We now focus on the algebraic structure of $A^{\prime \prime}$ in the case that $A$ is a lattice ordered algebra.

We say that $A$ is a lattice ordered algebra (or $l$-algebra, Riesz algebra), whenever $A$ is a vector lattice which is simultaneously an associative (but not necessarily commutative) algebra such that $x y \geq 0$ for all $0 \leq x, y \in A$

Received by the editors January 26, 1994.

1991 Mathematics Subject Classification. Primary 46A40, 46A20, 06F25, 13 J25.

Key words and phrases. Arens multiplication, $f$-algebra, almost $f$-algebra, $d$-algebra, second order dual, bidual, order continuous bidual. 
(equivalently, $|x y| \leq|x| \cdot|y|$ for all $x, y \in A$ ). In $A^{\prime \prime}$ a multiplication can be introduced (the Arens multiplication (see [2, 3]) which is accomplished in three steps. Given $x, y \in A, f \in A^{\prime}$ and $F, G \in A^{\prime \prime}$, we define $f \cdot x \in A^{\prime}$, $G \cdot f \in A^{\prime}$ and $F \cdot G \in A^{\prime \prime}$ by the equations

$$
\begin{aligned}
(f \cdot x)(y) & =f(x y), \\
(G \cdot f)(x) & =G(f \cdot x), \\
(F \cdot G)(f) & =F(G \cdot f) .
\end{aligned}
$$

It is straightforward to show that $A^{\prime \prime}$ is a lattice ordered algebra with respect to the Arens multiplication as described in (3) (see [7, Theorem 4.1]). Even if the original multiplication in $A$ is commutative, the Arens multiplication in $A^{\prime \prime}$ need not be. It is easily verified that $\sigma$ is an algebra homomorphism so when $A^{\prime}$ separates points of $A$ we can identify $A$ with the sub lattice algebra $\sigma(A)$ of $A^{\prime \prime}$.

The band of all order bounded, order continuous linear functionals on $A^{\prime}$ is denoted by $\left(A^{\prime}\right)_{n}^{\prime}$ and its disjoint complement in $A^{\prime \prime}$ by $\left(A^{\prime}\right)_{s}^{\prime}$, the band of all order bounded so-called singular functionals on $A^{\prime}$. Observe that

$$
A^{\prime \prime}=\left(A^{\prime}\right)_{n}^{\prime} \oplus\left(A^{\prime}\right)_{s}^{\prime},
$$

an order direct sum, as $A^{\prime \prime}$ is Dedekind complete. Because they are bands in a Dedekind complete vector lattice, $\left(A^{\prime}\right)_{n}^{\prime}$ and $\left(A^{\prime}\right)_{s}^{\prime}$ are themselves Dedekind complete vector lattices. It is an easy matter to show that $\left(A^{\prime}\right)_{n}^{\prime}$ is also an $l$-algebra with respect to the Arens multiplication ([7, Theorem 4.1]). Furthermore, $\sigma(A) \subset\left(A^{\prime}\right)_{n}^{\prime}$ which is almost obvious.

If $A$ happens to be commutative (which is the case for Archimedean almost $f$-algebras and $f$-algebras, but not for $d$-algebras; see [4, Theorem 2.15 and Example 4.1]), then $x^{\prime \prime} \cdot f=f \cdot x$ for all $x \in A, f \in A^{\prime}$. Indeed, this follows from

$$
\begin{aligned}
\left(x^{\prime \prime} \cdot f\right)(y) & =x^{\prime \prime}(f \cdot y)=(f \cdot y)(x)=f(y x)=f(x y) \\
& =(f \cdot x)(y)
\end{aligned}
$$

for all $y \in A$. Moreover, $x^{\prime \prime} \cdot F=F \cdot x^{\prime \prime}$ for all $x \in A, F \in A^{\prime \prime}$ in this situation. For the proof, note that

$$
\begin{aligned}
\left(x^{\prime \prime} \cdot F\right)(f) & =x^{\prime \prime}(F \cdot f)=(F \cdot f)(x)=F(f \cdot x) \\
& =F\left(x^{\prime \prime} \cdot f\right)=\left(F \cdot x^{\prime \prime}\right)(f)
\end{aligned}
$$

for all $f \in A^{\prime}$. These two properties will be used without further mention.

Let us recall the definitions of an $f$-algebra, almost $f$-algebra, and $d$-algebra (for a survey and most of the elementary properties we refer to [4]). The $l$-algebra $A$ is said to be an $f$-algebra whenever $x \wedge y=0$ and $0 \leq z \in A$ imply $x z \wedge y=z x \wedge y=0$ and an almost $f$-algebra if $x \wedge y=0$ implies $x y=0$. Any $f$-algebra is an almost $f$-algebra, but not conversely. Archimedean (almost) $f$-algebras are automatically commutative (and even associativity follows in case of $f$-algebras). If $A$ is an almost $f$-algebra, then $x x^{+} \geq 0$, and $x^{2} \geq 0$ for all $x \in A$. An $l$-algebra $A$ is a left $d$-algebra if $x \wedge y=0$ and $0 \leq z \in A$ imply $z x \wedge z y=0$. If $x \wedge y=0$ implies $x z \wedge y z=0$ for all $0 \leq z \in A$, then $A$ is termed a right $d$-algebra. An $l$-algebra that is both a left and a right $d$-algebra is simply called a $d$-algebra. Any $f$-algebra is a $d$-algebra, 
but not conversely. Almost $f$-algebras need not have the $d$-algebra property and vice versa. Archimedean $d$-algebras need not be commutative nor have positive squares. Archimedean $d$-algebras which are commutative or do have positive squares are almost $f$-algebras. Along the same line, that better algebraic properties force better order properties, note that Archimedean $d$-algebras and Archimedean almost $f$-algebras which are either unitary or semiprime (meaning that 0 is the only nilpotent element) are automatically $f$-algebras; for details of this we refer to [4].

In the next lines we will recollect some of the history of the order bidual of the various kinds of lattice ordered algebras. The first result in this direction is due to $\mathrm{B}$. de Pagter and the second author. They proved in the paper [7, Theorem 4.4] that the order continuous bidual $\left(A^{\prime}\right)_{n}^{\prime}$ of an Archimedean $f$-algebra $A$ is a Dedekind complete (hence Archimedean, commutative and Arens regular) $f$-algebra with respect to the Arens multiplication. In $\S 3$ of the present paper we will give two alternative proofs of this result which, in our opinion, are simpler and more straightforward. Some years after [7] was published, the second author, in [5, Theorem 2.8], proved that the entire second order dual $A^{\prime \prime}$ of an Archimedean $f$-algebra $A$ is an Archimedean $f$-algebra with respect to the Arens multiplication. This was also obtained independently by E. Scheffold in [11, Satz 2.2], by means of representations, for the class of Banach $f$-algebras. In [5] even more was shown: if $F \in A^{\prime \prime}, G \in\left(A^{\prime}\right)_{s}^{\prime}$, then $F \cdot G=G \cdot F=0$. This implies in particular that $F \cdot G \in\left(A^{\prime}\right)_{n}^{\prime}$ for all $F, G \in A^{\prime \prime}$ and furthermore that the Arens multiplication in $\left(A^{\prime}\right)_{s}^{\prime}$ is trivial.

In the survey paper [6, Section 8] it was conjectured by the second author that the order bidual $A^{\prime \prime}$ of an Archimedean almost $f$-algebra $A$ ( $d$-algebra $A)$ is ar Archimedean almost $f$-algebra ( $d$-algebra) with respect to the Arens multiplication. Recently, E. Scheffold presented a proof of the fact that the order continuous bidual $\left(A^{\prime}\right)_{n}^{\prime}$ of a Banach almost $f$-algebra $A$ is also a Banach almost $f$-algebra (equipped with the Arens multiplication), once more by means of representation theory [12, Theorem 3]. In $\S 2$ of the present paper we will provide the reader with an elementary proof of this result which is valid for the wider class of Archimedean almost $f$-algebras that do not carry a lattice norm. In fact, we will show that the whole order bidual $A^{\prime \prime}$ of an Archimedean almost $f$-algebra $A$ is also an Archimedean (whence commutative) almost $f$-algebra. Moreover, the results mentioned above for $f$-algebras carry over in this case: $F \cdot G=G \cdot F=0$ for all $F \in A^{\prime \prime}, G \in\left(A^{\prime}\right)_{s}^{\prime}$ and $F \cdot G \in\left(A^{\prime}\right)_{n}^{\prime}$ whenever $F, G \in A^{\prime \prime}$.

Finally, in [13, Theorem 2.1], E. Scheffold gives a complicated proof that the order continuous order bidual $\left(A^{\prime}\right)_{n}^{\prime}$ of a Banach $d$-algebra $A$ is a left Banach $d$-algebra. Difficulties arise for the class of $d$-algebras because Archimedean $d$-algebras need not be commutative. We are able to verify in $\S 4$ of this paper that whenever $A$ is an Archimedean $d$-algebra, $\left(A^{\prime}\right)_{n}^{\prime}$ is so too (i.e., $\left(A^{\prime}\right)_{n}^{\prime}$ is both a left and a right $d$-algebra). So far, we are not able to accomplish a similar result for the whole order bidual $A^{\prime \prime}$ of an Archimedean $d$-algebra $A$. The method of proof we developed in the case of (almost) $f$-algebras does not apply.

In the final paragraphs of this introduction we list some elementary facts on vector lattices that play a fundamental role in the sequel. First we quote the famous Riesz-Kantorovich theorem (see e.g. [1, Theorem 1.13]). If $A$ is a vec- 
tor lattice, then $A^{\prime}$ is a Dedekind complete vector lattice. Its lattice operations satisfy

$$
\begin{aligned}
& (g \vee h)(x)=\sup \{g(y)+h(z): x=y+z, 0 \leq y, z \in A\}, \\
& (g \wedge h)(x)=\inf \{g(y)+h(z): x=y+z, 0 \leq y, z \in A\}
\end{aligned}
$$

for all $g, h \in A^{\prime}, 0 \leq x \in A$.

Secondly, if $A$ is a vector lattice and $\sigma(A)=\left\{x^{\prime \prime}: x \in A\right\}$, then it is a well-known result of $\mathrm{H}$. Nakano that the order ideal

$$
I(\sigma(A))=\left\{F \in\left(A^{\prime}\right)_{n}^{\prime}:|F| \leq x^{\prime \prime} \text { for appropriate } 0 \leq x \in A\right\}
$$

generated by $\sigma(A)$ in $\left(A^{\prime}\right)_{n}^{\prime}$ is order dense in $\left(A^{\prime}\right)_{n}^{\prime}$ (see e.g. [10, Proposition 1.4.15]). This means that for every $0<F \in\left(A^{\prime}\right)_{n}^{\prime}$ there exists $G \in I(\sigma(A))$ satisfying $0<G \leq F$. Equivalently, there exist $0<F_{\alpha} \in I(\sigma(A))$ (say $0<$ $F_{\alpha} \leq x_{\alpha}^{\prime \prime}$ for some $\left.0<x_{\alpha} \in A\right)$ such that $F_{\alpha} \uparrow F$.

For $F \in A^{\prime \prime}$, the absolute kernel or null ideal $N_{F}$ of $F$ is defined by

$$
N_{F}=\left\{f \in A^{\prime}:|F|(|f|)=0\right\} \text {. }
$$

It is evident that $N_{F}=N_{|F|}=N_{F^{+}} \cap N_{F^{-}}$is an order ideal in $A^{\prime}$ that is (in general properly) contained in the null space of $F$. If $F \in\left(A^{\prime}\right)_{n}^{\prime}$, then $N_{F}$ is a band in $A^{\prime}$. The disjoint complement of $C_{F}=N_{F}^{d}$ is called the carrier of $F$ and is always a band in $A^{\prime}$.

For future reference we list some properties of the null ideal and the carrier in the next theorem.

Theorem 1.1. (i) (Nakano) If $G, H \in\left(A^{\prime}\right)_{n}^{\prime}$, then $G \perp H$ (i.e., $|G| \wedge|H|=0$ ) if and only if $C_{G} \subset N_{H}$ (and, by symmetry, if and only if $C_{H} \subset N_{G}$ ).

(ii) If $G \in\left(A^{\prime}\right)_{n}^{\prime}$, then $A^{\prime}=N_{G} \oplus C_{G}$ (a band decomposition).

(iii) If $H \in A^{\prime \prime}$, then $H \in\left(A^{\prime}\right)_{s}^{\prime}$ if and only if $N_{H}^{d d}=A^{\prime}$.

Proof. (i) See [1, Theorem 5.2] or [14, Theorem 90.6].

(ii) $[14$, Theorem 90.9].

(iii) For the only if part, a possible reference is [8, Theorem 50.4]. In order to show the converse, take $H \in A^{\prime \prime}$ such that $N_{H}$ is order dense in $A^{\prime}$. We may assume without loss of generality that $H \geq 0$, as $N_{H}=N_{|H|}$. Pick $0 \leq F \in\left(A^{\prime}\right)_{n}^{\prime}$ and observe that $N_{H} \subset N_{H \wedge F}$ implies $N_{H \wedge F}^{d d}=A^{\prime}$. On the other hand, $H \wedge F$ is order continuous, so $N_{H \wedge F}=N_{H \wedge F}^{d d}$. Combining these two equalities we find $N_{H \wedge F}=A^{\prime}$, so $H \wedge F=0$ for all $0 \leq F \in\left(A^{\prime}\right)_{n}^{\prime}$. This shows that $H \in\left(A^{\prime}\right)_{s}^{\prime}$.

Corollary 1.2. If $G, H \in\left(A^{\prime}\right)_{n}^{\prime}, G \perp H$, and $0 \leq f \in A^{\prime}$, then there exist $g, h \in$ $A^{\prime}$ such that $f=g+h, g \wedge h=0$, and $G(g)=0=H(h)$.

Proof. By Theorem 1.1(ii), there exist $g \in N_{G}$ and $h \in C_{G}$ such that $f=g+h$, and (clearly) $g \wedge h=0$. By Theorem 1.1(i), $C_{G} \subset N_{H}$, so $G(g)=0=H(h)$, as required.

\section{THE ORDER BIDUAL OF ALMOST $f$-ALGEBRAS}

In this section we will show first that the order continuous order bidual $\left(A^{\prime}\right)_{n}^{\prime}$ of an almost $f$-algebra $A$ is again an almost $f$-algebra (with respect to the Arens multiplication). Next we will show that $H \cdot G=0$ for all $H \in\left(A^{\prime}\right)_{s}^{\prime}$ and all $G \in\left(A^{\prime}\right)_{n}^{\prime}$. From this it will follow easily that $A^{\prime \prime}$ itself is an almost $f$-algebra. 
Proposition 2.1. Let $A$ be an almost $f$-algebra. If $0 \leq x \in A, 0 \leq G, H \in\left(A^{\prime}\right)_{n}^{\prime}$ satisfy $G \wedge H=0$ and $0 \leq G, H \leq x^{\prime \prime}$, then $G \cdot H=0$.

Proof. Choose $0 \leq f \in A^{\prime}$. By Corollary 1.2 there exist $g, h \in A^{\prime}$ such that $x^{\prime \prime} \cdot f=f \cdot x=g+h, g \wedge h=0$ and $G(g)=0=H(h)$. By the RieszKantorovich theorem,

$$
0=(g \wedge h)(x)=\inf \{g(y)+h(z): x=y+z, 0 \leq y, z \in A\} .
$$

If $\varepsilon>0$, the formula above gives $0 \leq y, z \in A$ such that $x=y+z$, and $g(y)+h(z)<\varepsilon$. (Note that $y$ and $z$ depend on $\varepsilon$.)

Now define the functionals $0 \leq G_{1}, H_{1} \in\left(A^{\prime}\right)_{n}^{\prime}$ by

$$
G_{1}=G \wedge(y-y \wedge z)^{\prime \prime}, \quad H_{1}=H \wedge(z-y \wedge z)^{\prime \prime} .
$$

Then

$$
\begin{aligned}
0 & \leq G-G_{1}=\left(G-(y-y \wedge z)^{\prime \prime}\right)^{+} \leq\left(x^{\prime \prime}-(y-y \wedge z)^{\prime \prime}\right)^{+} \\
& =y^{\prime \prime}+z^{\prime \prime}-(y-y \wedge z)^{\prime \prime} \leq 2 z^{\prime \prime}
\end{aligned}
$$

and similarly

$$
0 \leq H-H_{1} \leq 2 y^{\prime \prime}
$$

Observe now that

$$
(y-y \wedge z) \wedge(z-y \wedge z)=0
$$

so, because $A$ is an almost $f$-algebra,

$$
(y-y \wedge z) \cdot(z-y \wedge z)=0 \text {. }
$$

Hence,

$$
0 \leq G_{1} \cdot H_{1} \leq(y-y \wedge z)^{\prime \prime} \cdot(z-y \wedge z)^{\prime \prime}=0 .
$$

Now consider $\left(\left(G-G_{1}\right) \cdot H\right)(f)$. We have

$$
\begin{aligned}
0 & \leq\left(\left(G-G_{1}\right) \cdot H\right)(f)=\left(G-G_{1}\right)(H \cdot f) \leq\left(G-G_{1}\right)\left(x^{\prime \prime} \cdot f\right) \\
& =\left(G-G_{1}\right)(f \cdot x)=\left(G-G_{1}\right)(g+h) \\
& \leq G(g)+\left(G-G_{1}\right)(h) \leq 0+2 z^{\prime \prime}(h)=2 h(z)
\end{aligned}
$$

(where we use (1) and $G(g)=0$ ). Similarly,

$$
\begin{aligned}
0 & \leq\left(G_{1} \cdot\left(H-H_{1}\right)\right)(f) \leq\left(G \cdot\left(H-H_{1}\right)\right)(f) \leq\left(x^{\prime \prime} \cdot\left(H-H_{1}\right)\right)(f) \\
& =\left(\left(H-H_{1}\right) \cdot x^{\prime \prime}\right)(f)=\left(H-H_{1}\right)(f \cdot x)=\left(H-H_{1}\right)(g+h) \\
& \leq\left(H-H_{1}\right)(g)+H(h) \leq 2 y^{\prime \prime}(g)+0=2 g(y) .
\end{aligned}
$$

By (3), $G_{1} \cdot H_{1}=0$, so we easily check that

$$
G \cdot H=\left(G-G_{1}\right) \cdot H+G_{1} \cdot\left(H-H_{1}\right) .
$$

Therefore

$$
\begin{aligned}
0 & \leq(G \cdot H)(f)=\left(\left(G-G_{1}\right) \cdot H\right)(f)+\left(G_{1} \cdot\left(H-H_{1}\right)(f)\right. \\
& \leq 2 h(z)+2 g(y)<2 \varepsilon,
\end{aligned}
$$

where we use (4) and (5). Since $\varepsilon>0$ is arbitrary, we get $(G \cdot H)(f)=0$ for all $0 \leq f \in A^{\prime}$, so $G \cdot H=0$ and we are done.

The next result, which is an extension of Proposition 2.1 by means of standard order continuity arguments, was proved by E. Scheffold for Banach almost $f$-algebras via representation theory [12, Theorem 3$]$. 
Theorem 2.2. If $A$ is an almost $f$-algebra, then the order continuous order bidual $\left(A^{\prime}\right)_{n}^{\prime}$ is an almost $f$-algebra with respect to the Arens multiplication.

Proof. We have to show that if $0 \leq G, H \in\left(A^{\prime}\right)_{n}^{\prime}$ and $G \wedge H=0$, then $G \cdot H=0$. Let $I(\sigma(A))$ be the order ideal in $\left(A^{\prime}\right)_{n}^{\prime}$ generated by $\sigma(A)$; i.e.,

$$
I(\sigma(A))=\left\{K \in\left(A^{\prime}\right)_{n}^{\prime}:|K| \leq x^{\prime \prime} \text { for appropriate } 0 \leq x \in A\right\} .
$$

As observed in the introduction, $I(\sigma(A))$ is order dense in $\left(A^{\prime}\right)_{n}^{\prime}$, so there exist $G_{\alpha}, H_{\beta} \in I(\sigma(A))$ such that $0 \leq G_{\alpha} \uparrow G, 0 \leq H_{\beta} \uparrow H$ with, say, $0 \leq$ $G_{\alpha} \leq x_{\alpha}^{\prime \prime}, 0 \leq H_{\beta} \leq y_{\beta}^{\prime \prime}$ for some $0 \leq x_{\alpha}, y_{\beta} \in A$. Now $G \wedge H=0$ implies $G_{\alpha} \wedge H_{\beta}=0$ for all $\alpha, \beta$. Moreover,

$$
0 \leq G_{\alpha}, H_{\beta} \leq\left(x_{\alpha}+y_{\beta}\right)^{\prime \prime},
$$

so by Proposition 2.1 we have $G_{\alpha} \cdot H_{\beta}=0$ for all $\alpha, \beta$. Choose $0 \leq f \in A^{\prime}$. Since $0 \leq H_{\beta}(f \cdot x) \uparrow H(f \cdot x)$ for all $0 \leq x \in A$, we get $0 \leq H_{\beta} \cdot f \uparrow H \cdot f$. But each $G_{\alpha}$ is order continuous, so $0 \leq\left(G_{\alpha} \cdot H_{\beta}\right)(f) \uparrow\left(G_{\alpha} \cdot H\right)(f)$. This holds for all $0 \leq f \in A^{\prime}$, so $0 \leq G_{\alpha} \cdot H_{\beta} \uparrow G_{\alpha}$. Now $G_{\alpha} \cdot H_{\beta}=0$ for all $\beta$, showing that $G_{\alpha} \cdot H=0$ for all $\alpha$.

Since $0 \leq G_{\alpha} \uparrow G$, we have $0 \leq G_{\alpha}(H \cdot f) \uparrow G(H \cdot f)$ for all $0 \leq f \in A^{\prime}$ which implies $0 \leq G_{\alpha} \cdot H \uparrow G \cdot H$. Combined with $G_{\alpha} \cdot H=0$ for all $\alpha$ we finally may conclude that $G \cdot H=0$ and the theorem follows.

In order to prove that the whole order bidual of an almost $f$-algebra is again an almost $f$-algebra we need some preliminaries.

Lemma 2.3 (Cauchy inequality). If $A$ is an almost $f$-algebra and $0 \leq f \in A^{\prime}$, then

$$
|f(x y)| \leq \sqrt{f\left(x^{2}\right)} \sqrt{f\left(y^{2}\right)}
$$

for all $x, y \in A$.

Proof. Fix $x, y \in A$. Since $A$ is commutative and has positive squares, we have

for all $\alpha \in \mathbf{R}$. Hence

$$
(x-\alpha y)^{2}=x^{2}-2 \alpha x y+\alpha^{2} y^{2} \geq 0
$$

$$
f\left(x^{2}\right)-2 \alpha f(x y)+\alpha^{2} f\left(y^{2}\right) \geq 0
$$

for all real $\alpha$. Therefore, the discriminant of this quadratic polynomial in $\alpha$ satisfies

This gives the desired result.

$$
4 f(x y)^{2}-4 f\left(x^{2}\right) f\left(y^{2}\right) \leq 0
$$

Noting that $\left(A^{\prime}\right)_{n}^{\prime}$ is an almost $f$-algebra, and that each element $f$ of $A^{\prime}$ defines a linear functional on $\left(A^{\prime}\right)_{n}^{\prime}$ in standard fashion, we have the following consequence of Lemma 2.3.

Lemma 2.4. If $A$ is an almost $f$-algebra and $0 \leq f \in A^{\prime}$, then

$$
|(G \cdot H)(f)| \leq \sqrt{G^{2}(f)} \cdot \sqrt{H^{2}(f)}
$$

for all $G, H \in\left(A^{\prime}\right)_{n}^{\prime}$.

In particular, if $0 \leq G \in\left(A^{\prime}\right)_{n}^{\prime}$ and $0 \leq x \in A$ (take $H=x^{\prime \prime}$ ), we get

$$
0 \leq(G \cdot f)(x) \leq \sqrt{G^{2}(f)} \cdot \sqrt{f\left(x^{2}\right)}
$$

for all $0 \leq f \in A^{\prime}$. 
A corollary of Lemma 2.3 which we include for completeness but which we do not use subsequently is the following.

Corollary 2.5. Let $A$ be an almost $f$-algebra, $x \in A$ and $0 \leq f \in A^{\prime}$. Then $f \cdot x=0$ if and only if $f\left(x^{2}\right)=0$.

Incidentally, the latter follows also from the fact that $x^{2}=0$ if and only if $x y=0$ for all $y \in A$. In order to show that $x^{2}=0$ implies $x y=0$ for all $y$ we may assume that $x \geq 0$ and $y \geq 0$. Now it follows from $(y-n x)^{2} \geq 0$ that

$$
y^{2} \geq 2 n x y-n^{2} x^{2}=2 n x y \geq 0 \quad(n=1,2, \ldots) .
$$

By the Archimedean property, $x y=0$.

Another way to obtain this result is by observing that $(y-n x)(y-n x)^{+} \geq 0$.

$$
0 \leq x y-x y \wedge n x^{2} \leq x(y-y \wedge n x) \leq \frac{1}{n} y^{2}
$$

$(n=1,2, \ldots)$. Hence if $x^{2}=0$, then $0 \leq x y \leq n^{-1} y^{2} \quad(n=1,2, \ldots)$. The Archimedean property gives $x y=0$.

A more subtle result than Corollary 2.5 which is interesting in its own right and which we do need in the sequel reads as follows.

Proposition 2.6. Let $A$ be an almost $f$-algebra and $0 \leq f \in A^{\prime}$. Then

$$
(f \cdot x)^{+}=f \cdot x^{+}
$$

for all $x \in A$. In other words, $f \cdot A=\{f \cdot x: x \in A\}$ is a vector sublattice of $A^{\prime}$ (and $(f \cdot x)^{-}=f \cdot x^{-},|f \cdot x|=f \cdot|x|$ for all $\left.x \in A\right)$. Furthermore, the mapping $T_{f}: A \rightarrow A^{\prime}$ defined by $T_{f}(x)=f \cdot x$ is a lattice homomorphism.

Proof. Since $x^{+} y \geq x y$ for all $0 \leq y \in A$, we have

$$
\left(f \cdot x^{+}\right)(y)=f\left(x^{+} y\right) \geq f(x y)=(f \cdot x)(y)
$$

for all positive $y$, i.e., $f \cdot x^{+} \geq f \cdot x$. Also, $f \cdot x^{+} \geq 0$ showing that $f \cdot x^{+} \geq$ $(f \cdot x)^{+}$.

For the reverse inequality, take $0 \leq y \in A$ and put $g=(f \cdot x)^{+}$. Now the almost $f$-algebra property together with $x^{+} \wedge\left(x^{-} \wedge y\right)=0$ yields $x^{+}\left(x^{-} \wedge y\right)=$ 0 . Similarly, $\left(x^{-}-x^{-} \wedge y\right) \wedge\left(y-x^{-} \wedge y\right)=0$ results in $\left(x^{-}-x^{-} \wedge y\right) \cdot\left(y-x^{-} \wedge y\right)=$ 0 , so

$$
x^{-}\left(y-x^{-} \wedge y\right)=\left(x^{-} \wedge y\right) \cdot\left(y-x^{-} \wedge y\right)
$$

Hence,

$$
\begin{aligned}
g(y) & \geq g\left(y-x^{-} \wedge y\right) \geq(f \cdot x)\left(y-x^{-} \wedge y\right) \\
& =f\left(x y-x\left(x^{-} \wedge y\right)\right) \\
& =f\left(x^{+} y-x^{-} y-x^{+}\left(x^{-} \wedge y\right)+x^{-}\left(x^{-} \wedge y\right)\right) \\
& =f\left(x^{+} y-x^{-}\left(y-x^{-} \wedge y\right)\right) \\
& =f\left(x^{+} y-\left(x^{-} \wedge y\right)\left(y-x^{-} \wedge y\right)\right) \\
& \geq\left(f \cdot x^{+}\right)(y)-f\left(y^{2}\right) .
\end{aligned}
$$

Take $(1 / n) y$ in place of $y$ to get

$$
g(y) \geq\left(f \cdot x^{+}\right)(y)-\frac{1}{n} f\left(y^{2}\right) \quad(n=1,2, \ldots) .
$$


Consequently, $g(y) \geq\left(f \cdot x^{+}\right)(y)$. This holds for all $0 \leq y \in A$, so $(f \cdot x)^{+} \geq$ $f \cdot x^{+}$and the proof is complete.

We now come to a key result which seems to need the full force of Proposition 2.6. First notice that the adjoint $T_{f}^{\prime}: A^{\prime \prime} \rightarrow A^{\prime}$ of the above lattice homomorphism $T_{f}: A \rightarrow A^{\prime}$ is defined by

$$
\left(T_{f}^{\prime} G\right)(x)=G\left(T_{f} x\right)=G(f \cdot x)=(G \cdot f)(x)
$$

for all $x \in A, G \in A^{\prime \prime}$, so $T_{f}^{\prime} G=G \cdot f$ for all $G \in A^{\prime \prime}$.

Proposition 2.7. Let $A$ be an almost $f$-algebra, $0 \leq f \in A^{\prime}$ and $0 \leq H \in A^{\prime \prime}$. If $g \in A^{\prime}$ satisfies $0 \leq g \leq H \cdot f$, then there exists $\bar{K} \in A^{\prime \prime}$ such that $0 \leq K \leq H$ and $g=K \cdot f$. Hence, if $H \in\left(A^{\prime}\right)_{n}^{\prime}$, then $K \in\left(A^{\prime}\right)_{n}^{\prime}$ as well. In other words, $T_{f}^{\prime}$ is interval preserving (has the Maharam property).

Proof. By Proposition 2.6, if $x \in A$ and $f \cdot x=0$, then $f \cdot x^{+}=0$. Hence $0 \leq g\left(x^{+}\right) \leq(H \cdot f)\left(x^{+}\right)=H\left(f \cdot x^{+}\right)=0$ gives $g\left(x^{+}\right)=0$. Similarly $g\left(x^{-}\right)=$ 0 and hence $g(x)=0$. Accordingly we may define a linear functional $K$ on the vector sublattice $f \cdot A$ of $A^{\prime}$ by

$$
K(f \cdot x)=g(x)
$$

for all $x \in A$ (this definition makes sense by the above remarks). Clearly, $K$ is a positive linear functional on $f \cdot A$. Indeed, if $f \cdot x \geq 0$, then $f \cdot x=$ $(f \cdot x)^{+}=f \cdot x^{+}$gives $K(f \cdot x)=K\left(f \cdot x^{+}\right)=g\left(x^{+}\right) \geq 0$. Moreover, in this case $g \leq H \cdot f$ implies

$$
K(f \cdot x)=g\left(x^{+}\right) \leq(H \cdot f)\left(x^{+}\right)=H\left(f \cdot x^{+}\right)=H(f \cdot x),
$$

so $K$ is a positive linear functional on the vector sublattice $f \cdot A$ of $A^{\prime}$ satisfying $0 \leq K \leq H$ on $f \cdot A$. By the Hahn-Banach extension theorem for positive linear functionals (see e.g. [1, Theorem 2.2]) there exists a positive extension of $K$ to the whole of $A^{\prime}$ which is still dominated by $H$. If we denote this extension again by $K$, we have $0 \leq K \leq H$ on $A^{\prime}$ and for all $x \in A$ we have $(K \cdot f)(x)=K(f \cdot x)=g(x)$, so $g=K \cdot f$.

A slightly different approach to this result is obtained by using the result that the adjoint $T_{f}^{\prime}$ of the lattice homomorphism $T_{f}$ is interval preserving (see e.g. [1, Theorems 7.4 and 7.8]). Indeed, our proof of Proposition 2.7 is identical in concept with the proof of [1, Theorem 7.4] and both proofs use the Hahn-Banach theorem. Hence

$$
T_{f}^{\prime}[0, H]=\left[0, T_{f}^{\prime} H\right]=[0, H \cdot f],
$$

so if $0 \leq g \leq H \cdot f$, then $g=T_{f}^{\prime}(K)=K \cdot f$ for some $0 \leq K \leq H$.

Lemma 2.8. Let $A$ be an l-algebra and $G_{\alpha} \downarrow 0$ in $A^{\prime \prime}$. Then $G_{\alpha}^{2} \downarrow 0$ as well. Proof. Suppose that $0 \leq f \in A^{\prime}$. For $\beta$ fixed and all $\alpha$ with $G_{\alpha} \leq G_{\beta}$ we have

$$
0 \leq G_{\alpha}^{2}(f) \leq\left(G_{\alpha} \cdot G_{\beta}\right)(f)=G_{\alpha}\left(G_{\beta} \cdot f\right) \downarrow 0,
$$

from which the claim follows.

The next proposition plays a key role in the sequel. The proof is in the same spirit as the method used in the verification of [5, Proposition 2.2]. 
Proposition 2.9. Let $A$ be an almost $f$-algebra and $G_{\alpha} \downarrow 0$ in $\left(A^{\prime}\right)_{n}^{\prime}$. Then $F \cdot G_{\alpha} \downarrow 0$ for all $0 \leq F \in A^{\prime \prime}$.

Proof. Choose $0 \leq f \in A^{\prime}$. By Lemma 2.8 there exists a subsequence $\left\{G_{k}\right\}_{k=1}^{\infty}$ of the net $\left\{G_{\alpha}\right\}$ such that $G_{k}^{2}(f)<k^{-4}(k=1,2, \ldots)$. For $0 \leq x \in A$, Lemma 2.4 gives

$$
0 \leq \sum_{k=1}^{\infty}\left(G_{k} \cdot f\right)(x) \leq \sum_{k=1}^{\infty} \sqrt{G_{k}^{2}(f)} \cdot \sqrt{f\left(x^{2}\right)} \leq \sqrt{f\left(x^{2}\right)} \cdot \sum_{k=1}^{\infty} \frac{1}{k^{2}}<\infty .
$$

The functional $g: A^{+} \rightarrow \mathbf{R}$ defined by

$$
g(x)=\sum_{k=1}^{\infty}\left(G_{k} \cdot f\right)(x)
$$

is additive on $A^{+}$and has therefore (via standard arguments) a positive linear extension to the whole of $A$ ([14, Lemma 83.1]) which we shall denote by $g$ again.

We have to show that $\left(F \cdot G_{\alpha}\right)(f) \downarrow 0$. To this end we note that, for all positive integers $m$,

$$
F(g) \geq \sum_{k=1}^{m} F\left(G_{k} \cdot f\right)=\sum_{k=1}^{m}\left(F \cdot G_{k}\right)(f) .
$$

It follows that $\sum_{k=1}^{\infty}\left(F \cdot G_{k}\right)(f)$ is convergent and hence that $\left(F \cdot G_{k}\right)(f) \rightarrow 0$ as $k \rightarrow \infty$. It is now immediate that $\left(F \cdot G_{\alpha}\right)(f) \downarrow 0$, as required.

Our next result will lead directly to the final proof that $A^{\prime \prime}$ is an almost $f$-algebra whenever $A$ is so.

Theorem 2.10. Let $A$ be an almost f-algebra, $G \in\left(A^{\prime}\right)_{n}^{\prime}$ and $H \in\left(A^{\prime}\right)_{s}^{\prime}$. Then $H \cdot G=0$.

Proof. By splitting into positive and negative parts, if necessary, we may assume that $0 \leq G \in\left(A^{\prime}\right)_{n}^{\prime}$ and $0 \leq H \in\left(A^{\prime}\right)_{s}^{\prime}$. We have to show that $H(G \cdot f)=0$ for all $0 \leq f \in A^{\prime}$. Fix such an $f$ and put

$$
J=\left\{L \in\left(A^{\prime}\right)_{n}^{\prime}: L \geq 0,(H \cdot L)(f)=H(L \cdot f)=0\right\} .
$$

Clearly, $J$ is closed under addition and multiplication by positive scalars, and is a lattice ideal in the positive cone of $\left(A^{\prime}\right)_{n}^{\prime}$ (i.e., if $L_{1} \in J, 0 \leq L_{2} \leq L_{1}$, then $L_{2} \in J$ and if $L_{1}, L_{2} \in J$, then $\left.L_{1} \vee L_{2} \in J\right)$. The latter follows from

$$
0 \leq\left(H \cdot\left(L_{1} \vee L_{2}\right)\right)(f) \leq\left(H \cdot L_{1}\right)(f)+\left(H \cdot L_{2}\right)(f)=0 .
$$

We claim that $J$ is order dense in the positive cone of $\left(A^{\prime}\right)_{n}^{\prime}$. For this purpose we have to show that for each $0<M \in\left(A^{\prime}\right)_{n}^{\prime}$ there exists an element $L \in J$ satisfying $0<L \leq M$. If $(H \cdot M)(f)=0$, we may choose $L=M$, so suppose $(H \cdot M)(f)=H(M \cdot f)>0$. Since $N_{H}^{d d}=A^{\prime}$ there exists $g \in N_{H}$ (so $H(g)=0)$, such that $0<g \leq M \cdot f$. We note in passing that, by Theorem 1.1(iii), this makes full use of the fact that $H$ is singular. By Proposition 2.7, $g$ can be written in the form $g=L \cdot f$ with $L \in\left(A^{\prime}\right)_{n}^{\prime}$, and $0 \leq L \leq M$. Hence, $H(g)=H(L \cdot f)=0$, showing that $L \in J$. Because $g>0$ we have $L>0$ as well. 
Now consider the set

$$
J_{G}=\{L \in J: L \leq G\} .
$$

Since $J$ is a lattice ideal, $J_{G}$ is upwards directed. Moreover, $J_{G}$ is bounded above by $G$ in the Dedekind complete vector lattice $\left(A^{\prime}\right)_{n}^{\prime}$, so $G_{0}=\sup J_{G}$ exists in $\left(A^{\prime}\right)_{n}^{\prime}$ and $G_{0} \leq G$. Since, by Proposition 2.9,

$$
\left(H \cdot\left(G_{0}-L\right)\right)(f) \downarrow 0 \quad\left(L \in J_{G}\right),
$$

we have $\left(H \cdot G_{0}\right)(f)=0$, so $G_{0} \in J_{G}$. We assert that $G=G_{0}$. Suppose on the contrary that $G-G_{0}>0$. Since $J$ is order dense in the positive cone of $\left(A^{\prime}\right)_{n}^{\prime}$, there exists $L \in J$, with $0<L \leq G-G_{0}$. From this we obtain $0<L+G_{0} \leq G$. Together with $L+G_{0} \in J$ we get $L+G_{0} \in J_{G}$, so $L+G_{0} \leq G_{0}$ which is at variance with $L>0$. We have obtained a contradiction, so $G=G_{0} \in J$. Hence, $(H \cdot G)(f)=0$. This holds for all $0 \leq f \in A^{\prime}$, and therefore $H \cdot G=0$. This finishes the proof of the theorem.

Corollary 2.11. If $A$ is an almost $f$-algebra and $H \in\left(A^{\prime}\right)_{s}^{\prime}$, then $H \cdot f=0$ for all $f \in A^{\prime}$. Hence, $F \cdot H=0$ for all $F \in A^{\prime \prime}, H \in\left(A^{\prime}\right)_{s}^{\prime}$.

Proof. By Theorem 2.10, $H \cdot x^{\prime \prime}=0$ for all $x \in A$. Consequently $0=$ $\left(H \cdot x^{\prime \prime}\right)(f)=\left(x^{\prime \prime} \cdot H\right)(f)=x^{\prime \prime}(H \cdot f)=(H \cdot f)(x)$ for all $x \in A$, and $f \in A^{\prime}$. Therefore, $H \cdot f=0$ for all $f \in A^{\prime}$. It follows that $0=F(H \cdot f)=(F \cdot H)(f)$ for all $f \in A^{\prime}$, and all $F \in A^{\prime \prime}$; in other words, $F \cdot H=0$ for all $F \in A^{\prime \prime}$.

We are now in a position to prove the main result of this section.

Theorem 2.12. If $A$ is an almost $f$-algebra, then $A^{\prime \prime}$ is a (Dedekind complete and hence Archimedean) almost $f$-algebra with respect to the Arens multiplication.

Proof. Take $0 \leq F, G \in A^{\prime \prime}$ with $F \wedge G=0$ and decompose $F$ and $G$ according to the order direct sum $A^{\prime \prime}=\left(A^{\prime}\right)_{n}^{\prime} \oplus\left(A^{\prime}\right)_{s}^{\prime}$, so

$$
\begin{array}{ll}
F=F_{n}+F_{s} & \left(0 \leq F_{n} \in\left(A^{\prime}\right)_{n}^{\prime}, 0 \leq F_{s} \in\left(A^{\prime}\right)_{s}^{\prime}\right), \\
G=G_{n}+G_{s} & \left(0 \leq G_{n} \in\left(A^{\prime}\right)_{n}^{\prime}, 0 \leq G_{s} \in\left(A^{\prime}\right)_{s}^{\prime}\right) .
\end{array}
$$

Since $F_{n} \wedge G_{n}=0$ and, by Theorem 2.2, $\left(A^{\prime}\right)_{n}^{\prime}$ is an almost $f$-algebra, we have $F_{n} \cdot G_{n}=0$. By Theorem $2.10, F_{s} \cdot G_{n}=0$. By Corollary $2.11, F \cdot G_{s}=0$. It follows that $F \cdot G=0$ and we are done.

The proof of Theorem 2.12 shows that, for any $F, G \in A^{\prime \prime}$, we have $F \cdot G=$ $F_{n} \cdot G_{n}$. This fact, coupled with the observations that $\left(A^{\prime}\right)_{n}^{\prime}$ is commutative and closed under multiplication, makes the following two corollaries immediate.

Corollary 2.13. Let $A$ be an almost $f$-algebra. Then $F \cdot H=H \cdot F=0$ for all $F \in A^{\prime \prime}, H \in\left(A^{\prime}\right)_{s}^{\prime}$. In particular, $H^{2}=0$ for all $H \in\left(A^{\prime}\right)_{s}^{\prime}$.

Corollary 2.14. Let $A$ be an almost f-algebra. Then $F \cdot G \in\left(A^{\prime}\right)_{n}^{\prime}$ for all $F, G \in A^{\prime \prime}$.

\section{THE ORDER BIDUAL OF $f$-ALGEBRAS}

The second author and B. de Pagter [7, Theorem 4.4] proved that the order continuous bidual $\left(A^{\prime}\right)_{n}^{\prime}$ of an Archimedean $f$-algebra is an Archimedean $f$-algebra with respect to the Arens multiplication and the second author [5, 
Theorem 2.8] extended this to $A^{\prime \prime}$ itself (for the lattice normed case this last result was obtained independently by E. Scheffold in [11, Satz 2.2]). In this section we will show that these results can also be obtained by the methods of Proposition 2.1 and Theorem 2.10.

Theorem 3.1. Let $A$ be an $f$-algebra. Then $\left(A^{\prime}\right)_{n}^{\prime}$ is an $f$-algebra with respect to the Arens multiplication.

Proof. We will exploit the ideas used in the proof of Proposition 2.1. First, take $0 \leq G, H \in\left(A^{\prime}\right)_{n}^{\prime}$, and suppose that $G \wedge H=0$ and that $0 \leq G, H \leq x^{\prime \prime}$ for some $0 \leq x \in A$. Assume also that $F \in\left(A^{\prime}\right)_{n}^{\prime}$ satisfies $0 \leq F \leq x^{\prime \prime}$. We will show that $(F \cdot G) \wedge H=0$. To this end, fix $0 \leq f \in A^{\prime}$ and use Corollary 1.2 to obtain $g, h \in A^{\prime}$ such that

$$
f+f \cdot x=g+h, \quad \text { with } g \wedge h=0 \text { and } G(g)=0=H(h) .
$$

Suppose $\varepsilon>0$; because $g \wedge h=0$ there exist elements $0 \leq y, z \in A$ satisfying $x=y+z$, and $g(y)+h(z)<\varepsilon$. As before, put

$$
G_{1}=G \wedge(y-y \wedge z)^{\prime \prime} \text { and } H_{1}=H \wedge(z-y \wedge z)^{\prime \prime} \text {. }
$$

Then again,

$$
0 \leq G-G_{1} \leq 2 z^{\prime \prime} \text { and } 0 \leq H-H_{1} \leq 2 y^{\prime \prime} .
$$

Now $(y-y \wedge z) \wedge(z-y \wedge z)=0$ and $A$ is an $f$-algebra so $x(y-y \wedge z) \wedge(z-y \wedge z)=$ 0 . Hence,

$$
0 \leq\left(F \cdot G_{1}\right) \wedge H_{1} \leq x^{\prime \prime}(y-y \wedge z)^{\prime \prime} \wedge(z-y \wedge z)^{\prime \prime}=0
$$

gives $\left(F \cdot G_{1}\right) \wedge H_{1}=0$. We use the commutativity of $A$ to get

$$
\begin{aligned}
0 & \leq\left(F \cdot\left(G-G_{1}\right)\right)(f) \leq\left(x^{\prime \prime} \cdot\left(G-G_{1}\right)\right)(f)=\left(\left(G-G_{1}\right) \cdot x^{\prime \prime}\right)(f) \\
& =\left(G-G_{1}\right)(f \cdot x) \leq\left(G-G_{1}\right)(f+f \cdot x) \\
& =\left(G-G_{1}\right)(g+h) \leq G(g)+\left(G-G_{1}\right)(h) \leq 0+2 z^{\prime \prime}(h)=2 h(z) .
\end{aligned}
$$

Analogously,

$$
\begin{aligned}
0 & \leq\left(H-H_{1}\right)(f) \leq\left(H-H_{1}\right)(f+f \cdot x)=\left(H-H_{1}\right)(g+h) \\
& \leq\left(H-H_{1}\right)(g)+H(h) \leq 2 g(y) .
\end{aligned}
$$

It follows from

$$
\begin{aligned}
0 & \leq(F \cdot G) \wedge H \leq\left(F \cdot\left(G-G_{1}\right) \wedge H+\left(F \cdot G_{1}\right) \wedge\left(H-H_{1}\right)+\left(F \cdot G_{1}\right) \wedge H_{1}\right. \\
& \leq F \cdot\left(G-G_{1}\right)+\left(H-H_{1}\right)+0
\end{aligned}
$$

that

$$
\begin{aligned}
0 & \leq((F \cdot G) \wedge H)(f) \leq\left(F \cdot\left(G-G_{1}\right)\right)(f)+\left(H-H_{1}\right)(f) \\
& \leq 2 h(z)+2 g(y)<2 \varepsilon .
\end{aligned}
$$

The inequality $0 \leq(F \cdot G \wedge H)(f)<2 \varepsilon$ holds for all $\varepsilon>0$, so $((F \cdot G) \wedge H)(f)=0$. This being true for all $0 \leq f \in A^{\prime}$ we deduce $(F \cdot G) \wedge H=$ 0 . Similarly it is shown that $(G \cdot F) \wedge H=0$ (again we use the commutativity of $A$ ). Summarizing, we have shown so far that, if $0 \leq F, G, H \leq x^{\prime \prime}$ (for some $0 \leq x \in A)$ and $G \wedge H=0$, then $(F \cdot G) \wedge H=0=(G \cdot F) \wedge H$. In the general case when $F, G, H$ are arbitrary positive elements of $\left(A^{\prime}\right)_{n}^{\prime}$, with $G \wedge H=0$ we obtain $(F \cdot G) \wedge H=0=(G \cdot F) \wedge H$ from the fact that $I(\sigma(A))$ 
is order dense in $\left(A^{\prime}\right)_{n}^{\prime}$ and the same standard arguments that were used in the proof of Theorem 2.2.

Now, every $f$-algebra is an almost $f$-algebra so all the arguments used in $\S 2$ (Lemma 2.3 up to Corollary 2.11) and particularly Corollary 2.14 hold true. Suppose now that $G \wedge H=0$ in $A^{\prime \prime}$ and $0 \leq F \in A^{\prime \prime}$. Decompose all three of $F, G$ and $H$ into their order continuous and singular parts as follows.

$$
F=F_{n}+F_{s}, \quad G=G_{n}+G_{s}, \quad H=H_{n}+H_{s} .
$$

As in Corollary 2.14, $F \cdot G=F_{n} \cdot G_{n} \in\left(A^{\prime}\right)_{n}^{\prime}$, so $(F \cdot G) \wedge H_{s}=\left(F_{n} \cdot G_{n}\right) \wedge H_{s}=$ 0 . Since $G_{n} \wedge H_{n}=0$ and $\left(A^{\prime}\right)_{n}^{\prime}$ is an $f$-algebra, by Theorem 3.1, we have $F_{n} \cdot G_{n} \wedge H_{n}=0$. Taking all these observations into account we find

$$
(F \cdot G) \wedge H=\left(F_{n} \cdot G_{n}\right) \wedge\left(H_{n}+H_{s}\right)=\left(F_{n} \cdot G_{n}\right) \wedge H_{n}+\left(F_{n} \cdot G_{n}\right) \wedge H_{s}=0 .
$$

Similarly, $(G \cdot F) \wedge H=0$, so $A^{\prime \prime}$ is an $f$-algebra. We summarize these results in the next theorem.

Theorem 3.2. (cf. [15, Theorem 2.8]). If $A$ is an f-algebra, then $A^{\prime \prime}$ is a (Dedekind complete and hence Archimedean) $f$-algebra with respect to the Arens multiplication.

In the remainder of this section we present yet another (even simpler and essentially different) proof of the fact that the order continuous bidual $\left(A^{\prime}\right)_{n}^{\prime}$ of an Archimedean $f$-algebra is again an $f$-algebra. We first need a lemma.

Lemma 3.3. Let $A$ be an $f$-algebra, $0 \leq a \in A$ and $0 \leq f \in A^{\prime}$. Then

(i) $a x \leq x+a^{2} x$ for all $0 \leq x \in A$;

(ii) $f \cdot a \leq f+f \cdot a^{2}$.

Proof. (i) Multiplying the first term in

$$
(x-x \wedge a x) \wedge(a x-x \wedge a x)=0
$$

by $a$ we get

$$
(a x-a(x \wedge a x)) \wedge(a x-x \wedge a x)=0,
$$

which leads to

$$
a x=a(x \wedge a x) \vee(x \wedge a x) \leq a(x \wedge a x)+x \wedge a x \leq a^{2} x+x .
$$

(ii) By (i) and the positivity of $f$ we have

$$
(f \cdot a)(x)=f(a x) \leq f(x)+f\left(a^{2} x\right)=f(x)+\left(f \cdot a^{2}\right)(x)
$$

for all $0 \leq x \in A$. This is the desired result.

Now we are in a position to present an alternative proof of Theorem 3.1.

Theorem 3.4. The order continuous order bidual $\left(A^{\prime}\right)_{n}^{\prime}$ of an f-algebra $A$ is again an $f$-algebra (with respect to the Arens multiplication).

Proof. Observe first that Lemma 3.3(ii) implies that for each $0 \leq x \in A$ and $0 \leq G \in A^{\prime \prime}$ we have

$$
\left(x^{\prime \prime} \cdot G\right)(f)=G(f \cdot x) \leq G(f)+G\left(f \cdot x^{2}\right)=G(f)+\left(\left(x^{\prime \prime}\right)^{2} \cdot G\right)(f)
$$

for all $0 \leq f \in A^{\prime}$, so

$$
x^{\prime \prime} \cdot G \leq G+\left(x^{\prime \prime}\right)^{2} \cdot G .
$$


In this inequality replace $x^{\prime \prime}$ by $(1 / n) x^{\prime \prime}$ to obtain

$$
n\left(x^{\prime \prime} \cdot G\right) \leq n^{2} G+\left(x^{\prime \prime}\right)^{2} \cdot G \quad(n=1,2, \ldots) .
$$

Now take $0 \leq G, H \in A^{\prime \prime}$ such that $G \wedge H=0$. We claim that $(F \cdot G) \wedge H=$ 0 for all $0 \leq \bar{F} \in\left(A^{\prime}\right)_{n}^{\prime}$. First consider the case that $0 \leq F \leq x^{\prime \prime}$ for some $0 \leq x \in A$. By the above,

$$
\begin{aligned}
0 & \leq n\left(\left(x^{\prime \prime} \cdot G\right) \wedge H\right)=n\left(x^{\prime \prime} \cdot G\right) \wedge n H \\
& \leq n^{2} G \wedge n H+\left(\left(x^{\prime \prime}\right)^{2} \cdot G\right) \wedge n H \leq\left(x^{\prime \prime}\right)^{2} \cdot G \quad(n=1,2, \ldots) .
\end{aligned}
$$

Since $A^{\prime \prime}$ is Dedekind complete, and hence Archimedean, we find $\left(x^{\prime \prime} \cdot G\right) \wedge H=$ 0 . Consequently, $(F \cdot G) \wedge H=0$. If $F$ is an arbitrary positive element of $\left(A^{\prime}\right)_{n}^{\prime}$, there exist $F_{\alpha} \in I(\sigma(A))$ (say $\left.0 \leq F_{\alpha} \leq x_{\alpha}^{\prime \prime}\right)$ such that $0 \leq F_{\alpha} \uparrow F$. By the first part of this proof $\left(F_{\alpha} \cdot G\right) \wedge H=0$ for all $\alpha$. Since $0 \leq F_{\alpha} \cdot G \uparrow F \cdot G$ we find $(F \cdot G) \wedge H=0$.

Next consider $0 \leq F, G \in\left(A^{\prime}\right)_{n}^{\prime}$, and $0 \leq H \in A^{\prime \prime}$ such that $G \wedge H=0$. Since $x^{\prime \prime} \cdot G=G \cdot x^{\prime \prime}$ for all $x \in A$, we deduce from the earlier results that $\left(G \cdot x^{\prime \prime}\right) \wedge H=0$ for all $0 \leq x \in A$. With the same notation as above, $\left(G \cdot F_{\alpha}\right) \wedge$ $H=0$ for all $\alpha$. The order continuity of $G$ combined with $0 \leq F_{\alpha} \uparrow F$ immediately gives $0 \leq G \cdot F_{\alpha} \uparrow G \cdot F$ and $(G \cdot F) \wedge H=0$ follows.

The previous results make it clear that, if $G \wedge H=0$ in $\left(A^{\prime}\right)_{n}^{\prime}$, and $0 \leq F \in$ $\left(A^{\prime}\right)_{n}^{\prime}$, then

$$
(F \cdot G) \wedge H=0=(G \cdot F) \wedge H=0,
$$

so $\left(A^{\prime}\right)_{n}^{\prime}$ is an $f$-algebra.

From this point we can use the ideas of the first half of this section or follow the proof used in [5] to show that the whole order bidual $A^{\prime \prime}$ is an $f$-algebra whenever $A$ is. Our next theorem will describe yet another approach.

Theorem 3.5. The order bidual $A^{\prime \prime}$ of an $f$-algebra $A$ is also an $f$-algebra.

Proof. We have shown in the proof of Theorem 3.4 that $(F \cdot G) \wedge H=0$ whenever $0 \leq F \in\left(A^{\prime}\right)_{n}^{\prime}$ and $0 \leq G, H \in A^{\prime \prime}$ satisfy $G \wedge H=0$. We will show that the previous hypotheses also lead to $(G \cdot F) \wedge H=0$. Indeed apply Lemma 3.3(ii) to the positive elements $F$ of $\left(A^{\prime}\right)_{n}^{\prime}$ and $f$ of $A^{\prime}$, considered as a linear functional on $\left(A^{\prime}\right)_{n}^{\prime}$, to obtain

$$
F \cdot f \leq f+F^{2} \cdot f
$$

for all $0 \leq f \in A^{\prime}$. Since $0 \leq G \in A^{\prime \prime}$ we have

$$
(G \cdot F)(f)=G(F \cdot f) \leq G(f)+G\left(F^{2} \cdot f\right)=G(f)+\left(G \cdot F^{2}\right)(f)
$$

for all $0 \leq f \in A^{\prime}$, so

$$
G \cdot F \leq G+G \cdot F^{2} .
$$

Since $G \wedge H=0$, we have $(G \cdot F) \wedge H \leq G \wedge H+\left(G \cdot F^{2}\right) \wedge H=\left(G \cdot F^{2}\right) \wedge H$. Replacing $F$ with $(1 / n) F$ leads to

$$
(G \cdot F) \wedge H \leq(1 / n)\left(G \cdot F^{2}\right) \wedge H \quad(n=1,2, \ldots) .
$$

Because $A$ is Archimedean, $(G \cdot F) \wedge H=0$.

It was shown in Proposition 2.2 of [5] that $F \in\left(A^{\prime}\right)_{n}^{\prime}$ and $G \in A^{\prime \prime}$ imply $G \cdot F \in\left(A^{\prime}\right)_{n}^{\prime}$. (The proof is technical, but elementary, and can be simplified to look very much like the proof of Proposition 2.9.) Take $0 \leq G \in\left(A^{\prime}\right)_{s}^{\prime}, 0 \leq$ 
$F \in\left(A^{\prime}\right)_{n}^{\prime}$. By the result just cited $G \cdot F \in\left(A^{\prime}\right)_{n}^{\prime}$. Also $G \wedge H=0$ for all $0 \leq$ $H \in\left(A^{\prime}\right)_{n}^{\prime}$, so by the earlier observations $G \cdot F \wedge H=0$ for all $0 \leq H \in\left(A^{\prime}\right)_{n}^{\prime}$, i.e., $G \cdot F \in\left(A^{\prime}\right)_{s}^{\prime}$. Hence, $G \cdot F=0$ for all $0 \leq G \in\left(A^{\prime}\right)_{s}^{\prime}$, and $0 \leq F \in\left(A^{\prime}\right)_{n}^{\prime}$. This is precisely the outcome of Theorem 2.10. For the rest of the proof, follow the arguments from Corollary 2.11, Corollary 2.14 and Theorem 3.2.

\section{THE ORDER CONTINUOUS ORDER BIDUAL OF $d$-ALGEBRAS}

Recently, E. Scheffold showed [13, Theorem 2.1] that the order continuous order bidual $\left(A^{\prime}\right)_{n}^{\prime}$ of a Banach $d$-algebra is a left Banach $d$-algebra with respect to the Arens multiplication. He was not able to show that $\left(A^{\prime}\right)_{n}^{\prime}$ is also a right Banach $d$-algebra, the main reason for this being that Archimedean $d$-algebras need not be commutative (see e.g. [4, Example 4.1]). In the present section we will generalize Scheffold's results considerably by showing that the order continuous order bidual $\left(A^{\prime}\right)_{n}^{\prime}$ of an Archimedean $d$-algebra is an Archimedean (and even Dedekind complete) $d$-algebra with respect to the Arens multiplication. The $d$-algebra $A$ we will consider need not carry a lattice norm and we will show that $\left(A^{\prime}\right)_{n}^{\prime}$ is not only a left but also a right $d$-algebra. Our proofs will be completely different from Scheffold's proofs and, we believe, more elementary.

So far we are unable to prove or disprove the corresponding result for the whole order bidual $A^{\prime \prime}$ of an Archimedean $d$-algebra $A$, the main reasons being that $d$-algebras need not be commutative nor need they have positive squares. (For an example of the latter, see [4, Example 4.1].) This means that the method used in $\S 2$ for almost $f$-algebras (and in particular Lemma 2.3 ) does not work in this case. In the proof of the next theorem we are able to use the same basic methods that were developed in the proof of Proposition 2.1 and Theorem 3.1.

Theorem 4.1. Let $A$ be a d-algebra. Then $\left(A^{\prime}\right)_{n}^{\prime}$ is a d-algebra with respect to the Arens multiplication.

Proof. We have to show that if $0 \leq F, G, H \in\left(A^{\prime}\right)_{n}^{\prime}$ and $G \wedge H=0$, then $(F \cdot G) \wedge(F \cdot H)=0$ (so $\left(A^{\prime}\right)_{n}^{\prime}$ is a left $d$-algebra) and $(G \cdot F) \wedge(H \cdot F)=0$ (so $\left(A^{\prime}\right)_{n}^{\prime}$ is a right $d$-algebra). We may assume that $0 \leq F, G, H \leq x^{\prime \prime}$ for some $0 \leq x \in A$. The standard order continuity arguments (use that $I(\sigma(A)$ ) is order dense in $\left.\left(A^{\prime}\right)_{n}^{\prime}\right)$ then yield the general result. Take $0 \leq f \in A^{\prime}$ and define the positive linear functional $f * x$ by

$$
(f * x)(y)=f(y x)
$$

for all $y \in A$ (the dual functional of $f \cdot x$ ). Of course $f \cdot x=f * x$ whenever $A$ is commutative, but Archimedean $d$-algebras need not be! By Corollary 1.2 again, there exist $0 \leq g, h \in A^{\prime}$ with $g \wedge h=0$, and $G(g)=0=H(h)$ such that

$$
f \cdot x+f * x=g+h .
$$

Suppose $\varepsilon>0$; since $g \wedge h=0$ there exist elements $0 \leq y, z \in A$ with $x=y+z$, and $g(y)+h(z)<\varepsilon$. As before we define $G_{1}=G \wedge(y-y \wedge z)^{\prime \prime}$, and $H_{1}=H \wedge(z-y \wedge z)^{\prime \prime}$. Again we have $0 \leq G-G_{1} \leq 2 z^{\prime \prime}, 0 \leq H-H_{1} \leq 2 y^{\prime \prime}$. It follows from

$$
\begin{aligned}
0 & \leq\left(G_{1} \cdot F\right) \wedge\left(H_{1} \cdot F\right) \leq\left(G_{1} \cdot x^{\prime \prime}\right) \wedge\left(H_{1} \cdot x^{\prime \prime}\right) \\
& \leq\left((y-y \wedge z)^{\prime \prime} \cdot x^{\prime \prime}\right) \wedge\left((z-y \wedge z)^{\prime \prime} \cdot x^{\prime \prime}\right)=0
\end{aligned}
$$


(here we use the right $d$-algebra property in $A$ ) that

$$
\left(G_{1} \cdot F\right) \wedge\left(H_{1} \cdot F\right)=0 .
$$

Observe that

$$
\left(x^{\prime \prime} \cdot f\right)(y)=x^{\prime \prime}(f \cdot y)=(f \cdot y)(x)=f(y x)=(f * x)(y)
$$

for all $y \in A$, so $x^{\prime \prime} \cdot f=f * x$. Hence,

$$
\begin{aligned}
0 & \leq\left(\left(G-G_{1}\right) \cdot F\right)(f) \leq\left(\left(G-G_{1}\right) \cdot x^{\prime \prime}\right)(f)=\left(G-G_{1}\right)\left(x^{\prime \prime} \cdot f\right) \\
& =\left(G-G_{1}\right)(f * x) \leq\left(G-G_{1}\right)(f \cdot x+f * x) \\
& =\left(G-G_{1}\right)(g+h) \leq G(g)+\left(G-G_{1}\right)(h) \leq 0+2 z^{\prime \prime}(h)=2 h(z) .
\end{aligned}
$$

Analogously,

$$
0 \leq\left(\left(H-H_{1}\right) \cdot F\right)(f) \leq 2 g(y) .
$$

Now,

$$
\begin{aligned}
(G \cdot F) \wedge(H \cdot F) & =\left(G-G_{1}+G_{1}\right) \cdot F \wedge\left(H-H_{1}+H_{1}\right) \cdot F \\
& \leq\left(G-G_{1}\right) \cdot F+\left(H-H_{1}\right) \cdot F+\left(G_{1} \cdot F\right) \wedge\left(H_{1} \cdot F\right) \\
& =\left(G-G_{1}\right) \cdot F+\left(H-H_{1}\right) \cdot F
\end{aligned}
$$

shows that

$$
\begin{aligned}
0 & \leq((G \cdot F) \wedge(H \cdot F))(f) \leq\left(\left(G-G_{1}\right) \cdot F\right)(f)+\left(\left(H-H_{1}\right) \cdot F\right)(f) \\
& \leq 2 h(z)+2 g(y)<2 \varepsilon .
\end{aligned}
$$

Hence, since $\varepsilon>0$ is arbitrary, we get $((G \cdot F) \wedge(H \cdot F))(f)=0$ for all $0 \leq$ $f \in A^{\prime}$, so $(G \cdot F) \wedge(H \cdot F)=0$. Similarly, $(F \cdot G) \wedge(F \cdot H)=0$ and the proof is complete.

Another way of obtaining the result of Theorem 4.1 is by means of the theory of lattice homomorphisms. Indeed, if $A$ is an $l$-algebra, then $A$ is a $d$-algebra if and only if for each $0 \leq x \in A$ the right multiplication, $R_{x}$, by $x$ (defined by $R_{x}(y)=y x$ for all $\left.x \in A\right)$ and the left multiplication, $L_{x}$, by $x \quad\left(L_{x}(y)=x y\right.$ for all $y \in A$ ) are lattice homomorphisms of $A$. Hence, the first adjoints $R_{x}^{\prime}$ and $L_{x}^{\prime}$ are interval preserving and consequently the second adjoints $R_{x}^{\prime \prime}$ and $L_{x}^{\prime \prime}$ are lattice homomorphisms of $A^{\prime \prime}$ (for details, see [1, Theorems 7.4, 7.8 and 7.9]).

We claim that $R_{x}^{\prime \prime}(G)=G \cdot x^{\prime \prime}$ for all $G \in A^{\prime \prime}$ (so $R_{x}^{\prime \prime}=R_{x^{\prime \prime}}$ in $A^{\prime \prime}$ ). Indeed, if $f \in A^{\prime}$, then

$$
\left(R_{x}^{\prime} f\right)(y)=f\left(R_{x} y\right)=f(y x)=(f \cdot y)(x)=x^{\prime \prime}(f \cdot y)=\left(x^{\prime \prime} \cdot f\right)(y)
$$

for all $y \in A$ implies $R_{x}^{\prime} f=x^{\prime \prime} \cdot f$. Consequently, if $G \in A^{\prime \prime}$, then

$$
\left(R_{x}^{\prime \prime} G\right)(f)=G\left(R_{x}^{\prime} f\right)=G\left(x^{\prime \prime} \cdot f\right)=\left(G \cdot x^{\prime \prime}\right)(f)
$$

for all $f \in A^{\prime}$ yields $R_{x}^{\prime \prime}(G)=G \cdot x^{\prime \prime} \quad\left(x \in A, G \in A^{\prime \prime}\right)$. Similarly, $L_{x}^{\prime} f=f \cdot x$ $\left(x \in A, f \in A^{\prime}\right)$ and $L_{x}^{\prime \prime}(G)=x^{\prime \prime} \cdot G\left(x \in A, G \in A^{\prime \prime}\right)$, so $L_{x}^{\prime \prime}=L_{x^{\prime \prime}}$ in $A^{\prime \prime}$.

Now take $0 \leq x \in A$ and $0 \leq F, G \in A^{\prime \prime}$ such that $F \wedge G=0$. Since $L_{x}^{\prime \prime}$ is a lattice homomorphism of $A^{\prime \prime}$, we get

$$
\left(x^{\prime \prime} \cdot F\right) \wedge\left(x^{\prime \prime} \cdot G\right)=L_{x}^{\prime \prime}(F) \wedge L_{x}^{\prime \prime}(G)=0 .
$$

By the standard order continuity arguments we find that for all $0 \leq H \in\left(A^{\prime}\right)_{n}^{\prime}$ we have $(H \cdot F) \wedge(H \cdot G)=0$. In particular, $\left(A^{\prime}\right)_{n}^{\prime}$ is a left $d$-algebra. (In fact 
we proved a slightly stronger result.) If $F \wedge G=0$ in $\left(A^{\prime}\right)_{n}^{\prime}$, then as above $\left(F \cdot x^{\prime \prime}\right) \wedge\left(G \cdot x^{\prime \prime}\right)=R_{x}^{\prime \prime}(F) \wedge R_{x}^{\prime \prime}(G)=0$. This implies easily that $(F \cdot H) \wedge$ $(G \cdot H)=0$, so $\left(A^{\prime}\right)_{n}^{\prime}$ is also a right $d$-algebra. (But this time we need the order continuity of $F$ and $G$ !)

As observed before, we have not been able to prove that the whole second order dual $A^{\prime \prime}$ of a $d$-algebra $A$ is a $d$-algebra as well, mainly because we have not found a way to handle the singular part of $A^{\prime \prime}$. We have observed already that $A$ need not be commutative, neither need $A$ have positive squares. If we impose one of these additional conditions on $A$ the situation becomes simple.

Theorem 4.2. Let $A$ be a d-algebra which is commutative or has positive squares. Then $A^{\prime \prime}$ is a Dedekind complete, hence Archimedean, $d$-algebra with respect to the Arens multiplication. Moreover, $A^{\prime \prime}$ is commutative and its squares are positive.

Proof. By [4, Theorem 4.5] each of the conditions on $A$ implies that $A$ is an almost $f$-algebra. By Corollary $2.14, F \cdot G=F_{n} \cdot G_{n}$ for all $F, G \in A^{\prime \prime}$, where $F_{n}$ and $G_{n}$ denote the order continuous parts of $F$ and $G$, respectively. Now assume that $G \wedge H=0$ in $A^{\prime \prime}$ and $0 \leq F \in A^{\prime \prime}$. Decompose all three of $F, G, H$ into their order continuous and singular components as follows.

$$
F=F_{n}+F_{s}, \quad G=G_{n}+G_{s}, \quad H=H_{n}+H_{s},
$$

according to the band decomposition $A^{\prime \prime}=\left(A^{\prime}\right)_{n}^{\prime} \oplus\left(A^{\prime}\right)_{s}^{\prime}$. Since, by Theorem 4.1, $\left(A^{\prime}\right)_{n}^{\prime}$ is a $d$-algebra and $G_{n} \wedge H_{n}=0$, we have

$$
\left(F_{n} \cdot G_{n}\right) \wedge\left(F_{n} \cdot H_{n}\right)=0=\left(G_{n} \cdot F_{n}\right) \wedge\left(H_{n} \cdot F_{n}\right) .
$$

By Corollary 2.14, $F \cdot G=F_{n} \cdot G_{n}, F \cdot H=F_{n} \cdot H_{n}$, so $(F \cdot G) \wedge(F \cdot H)=0$. Similarly, $(G \cdot F) \wedge(H \cdot F)=0$, so $A^{\prime \prime}$ is a $d$-algebra. By Theorem $2.12, A^{\prime \prime}$ is an Archimedean almost $f$-algebra; in particular $A^{\prime \prime}$ is commutative and its squares are positive.

\section{ACKNOWLEDGMENTS}

We thank J. J. Grobler for pointing out to us that we could use the facts that the adjoint of a lattice homomorphism is interval preserving (in the different approach in the proof of Proposition 2.7) and that the bi-adjoint of a lattice homomorphism is again a lattice homomorphism (in the alternative proof of Theorem 4.1). We also thank the referee for providing the bibliographical information for [13].

\section{REFERENCES}

1. C. D. Aliprantis and O. Burkinshaw, Positive operators, Academic Press, Orlando, FL, 1985.

2. R. Arens, Operations induced in function classes, Monatsh. Math. 55 (1951), 1-19.

3. _ The adjoint of a bilinear operation, Proc. Amer. Math. Soc. 2 (1951), 839-848.

4. S. J. Bernau and C. B. Huijsmans, Almost f-algebras and d-algebras, Math. Proc. Cambridge Philos. Soc. 107 (1990), 287-308.

5. C. B. Huijsmans, The order bidual of lattice ordered algebras. II, J. Operator Theory 22 (1989), 277-290.

6. __ Lattice ordered algebras and f-algebras: A survey, Studies in Economic Theory 2, Positive Operators, Riesz Spaces and Economics (C. D. Aliprantis, K. C. Border and W. A. J. Luxemburg, eds.), Springer, Berlin, 1991, pp. 151-169. 
7. C. B. Huijsmans and B. de Pagter, The order bidual of lattice ordered algebras, J. Funct. Anal. 59 (1984), 41-64.

8. W. A. J. Luxemburg, Notes on Banach function spaces. XVa, Indag. Math. 27 (1965), 415-429.

9. W. A. J. Luxemburg and A. C. Zaanen, Riesz spaces. I, North-Holland, Amsterdam, 1971.

10. P. Meyer-Nieberg, Banach lattices, Universitext, Springer, Berlin, 1991.

11. E. Scheffold, Der Bidual von F-Banachverbandsalgebren, Acta Sci. Math. 55 (1991), 167179.

12. _ Ueber den ordnungsstetigen Bidual von FF-Banachverbandsalgebren, Arch. Math. 60 (1993), 473-477.

13. _ Ueber Bimorphismen und das Arens-Produkt bei kommutativen D-Banachverbandsalgebren, Rev. Roumaine Math. Pures Appl. 39 (1994), 259-270.

14. A. C. Zaanen, Riesz spaces. II, North-Holland, Amsterdam, 1983.

Department of Mathematical Sciences, University of TeXas at El Paso, El Paso, Texas 79968-0514

E-mail address: simon@math.utep.edu

Department of Mathematics, Rijksuniversiteit Leiden, P.O. Box 9512, 2300 RA Leiden, THE NETHERLANDS

E-mail address: chuijsmans@rulcri.leidenuniv.nl 